Nephron 1978;20:181

\title{
Summary or Expansion?
}

During 1977, 365 medical publications will drop through my letter-box. Many will be general medical journals containing articles of renal interest and 54 will be issues of Nephron and the other renal journals; in 1978 they will number 72. Twice a year, with a hefty thump, 700 pages of Trans ASAIO or Proc EDTA join them on the doormat. No neglect of my duties, no courses in rapid reading could enable me to wade through this morass of information and verbiage. The best I can hope for is to scan the summaries and pick out the few articles I have time to read, file or even read and file.

This honest endeavour is frustrated by authors who reproduce the whole of their results section in the summary and often cram in the tables and discussions to boot. Means, standard errors, $p$ values, correlation coefficients jostle one another in line after line of telegraphic English and incomprehensible contractions. It is time to call a halt. With the authority of the Editor, I commend to all who are composing summaries for articles in Nephron the following rules:

(1) State the main message of the paper

clearly and briefly. Leave the side issues and nuances for the discussion.

Do not express in numbers what you can put in plain English. Most doctors are literate, few think numerically.

Put no more data in the summary than needed to commend the paper. If you studied 100 patients, say so. If the renal plasma flow fell substantially and significantly say just that; put the ' $597 \pm$ 11.2 (SD) to $534 \pm 13.6(\mathrm{p}<0.0001)^{\prime}$ in the results section where it belongs.

Use no contractions unless they are universally understood. GFR and CSF may just qualify but SOB is 'short of breath' in England and 'son of a bitch' in the United States.

Keep it short. Twelve lines of Nephron summary can be read comfortably in the one minute which nephrologists, other than your wife, old cronies and deadly rivals are likely to allot it. If you must repeat yourself, do it in a 'conclusions' section at the end of the paper -and send the extra page charges!

Pนoí.D.N.S.Kerr 